\title{
On the evolution of multiple low mass planets embedded in a circumbinary disc
}

\author{
A. Pierens and R. P. Nelson
}

Astronomy Unit, Queen Mary, University of London, Mile End Rd, London E1 4NS, UK

e-mail: a.pierens@qmul.ac.uk

Received 12 October 2007 / Accepted 5 December 2007

\section{ABSTRACT}

\begin{abstract}
Context. Previous work has shown that the tidal interaction between a binary system and a circumbinary disc leads to the formation of a large inner cavity in the disc. Subsequent formation and inward migration of a low mass planet causes it to become trapped at the cavity edge, where it orbits until further mass growth or disc dispersal. The question of how systems of multiple planets in circumbinary discs evolve has not yet been addressed.

Aims. We present the results of hydrodynamic simulations of multiple low mass planets embedded in a circumbinary disc. The aim is to examine their long term evolution as they approach and become trapped at the edge of the tidally truncated inner cavity. Methods. A grid-based hydrodynamics code was used to compute simulations of 2D circumbinary disc models with embedded planets. The 3D evolution of the planet orbits was computed, and inclination damping due to the disc was calculated using prescribed forces. We present a suite of simulations which study the evolution of pairs of planets migrating in the disc. We also present the results of hydrodynamic simulations of five-planet systems, and study their long term evolution after disc dispersal using a $N$-body code. Results. For the two-planet simulations we assume that the innermost planet has migrated to the edge of the inner cavity and remains trapped there, and study the subsequent evolution of the system as the outermost planet migrates inward. We find that the outcomes largely depend on the mass ratio $q=m_{\mathrm{i}} / m_{\mathrm{o}}$, where $m_{\mathrm{i}}\left(m_{\mathrm{o}}\right)$ is the mass of the innermost (outermost) planet. For $q<1$, planets usually undergo dynamical scattering or orbital exchange. For values of $q>1$ the systems reach equilibrium configurations in which the planets are locked into mean motion resonances, and remain trapped at the edge of the inner cavity without further migration. Most simulations of five-planet systems we performed resulted in collisions and scattering events, such that only a single planet remained in orbit about the binary. In one case however, a multiplanet resonant system was found to be dynamically stable over long time scales, suggesting that such systems may be observed in planet searches focussed on close binary systems.
\end{abstract}

Key words. accretion, accretion disks - planetary systems: formation - stars: binaries: close - hydrodynamics - methods: numerical

\section{Introduction}

At the time of writing approximately 250 extrasolar planets have been discovered, of which about 30 are members of binary or multiple stellar systems (Eggenberger et al. 2004; Mugrauer et al. 2007). Most of the planets found in binaries orbit around one of the stellar component in so-called S-type orbits, and the majority of binaries harbouring planets have orbital separations $a_{\mathrm{b}} \geq 100 \mathrm{AU}$. There are, however, exceptions to these cases. The short period binary systems Gliese 86, $\gamma$ Cephei and HD 41004 have $a_{\mathrm{b}} \sim 20 \mathrm{AU}$, and contain planets orbiting at 1-2 AU from the central star (Eggenberger et al. 2004; Mugrauer \& Neuhauser 2005). Although there are no known planets which orbit around both stellar companions in a binary system consisting of main sequence stars (i.e. circumbinary planets on so-called P-type orbits), there are two systems which indicate that the formation of circumbinary planets is feasible. The first is the circumbinary planet with mass $m_{\mathrm{p}}=2.5 M_{J}$ and orbital radius $23 \mathrm{AU}$ observed in the radio pulsar PSR 1620-26 (Sigurdsson et al. 2003). The second is the $m_{\mathrm{p}}=2.44 M_{J}$ planet orbiting about the system composed of the star HD 202206 and its 17.4 $M_{J}$ brown dwarf companion (Udry et al. 2002). The lack of observed circumbinary planets is probably due to the fact that short period binaries are usually rejected from observational surveys.

The observation of planets in binary systems is consistent with detections of circumstellar discs in binary systems. Several circumbinary discs have been detected around spectroscopic binaries such as DQ Tau, AK Sco, and GW Ori. The circumbinary disc around GG Tau has been directly imaged (Dutrey et al. 1994), revealing the presence of a tidally truncated inner cavity generated by the central binary. The existence of these circumbinary discs opens up the possibility of circumbinary planets forming. Combining the observations of circumbinary discs, with the fact that $\sim 50 \%$ of solar-type stars are members of binaries (Duquennoy \& Mayor 1991), suggests that circumbinary planets are probably common.

To date there has been a relatively modest amount of theoretical work examining planet formation in binary systems. Results from previous studies suggest that planetesimal accretion should be possible in regions of both circumstellar (Marzari \& Scholl 2000; Thebault et al. 2006) and circumbinary discs (Moriwaki \& Nakagawa 2004; Scholl et al. 2007). Quintana \& Lissauer (2006) simulated the late stages of terrestial planet formation in circumbinary discs. They found that planetary systems similar to those around single stars can be formed around binaries, provided the ratio of the binary apocentre distance to planetary orbit is $\leq 0.2$. In general binaries with larger maximum separations lead to planetary systems with fewer planets.

The evolution of a low mass planetary core embedded in a circumbinary disc was investigated recently by Pierens \& Nelson (2007) (hereafter referred to as Paper I). This work examined the migration and long term orbital evolution of planets with masses 
of $m_{\mathrm{p}}=5,10$ and $20 M_{\oplus}$ under the action of disc torques. It was found that the inward drift of a planet undergoing type I migration is stopped at the edge of the cavity formed by the binary. This halting of migration is due to positive corotation torques operating which can counterbalance negative Lindblad torques. Such an effect is known to be at work in accretion disc regions where there is a strong positive gradient of the surface density (Masset et al. 2006). Interestingly, Pierens \& Nelson (2007) showed that the stopping of migration in circumbinary discs occurs in a region of long-term dynamical stability, suggesting that such planets may be able to survive there over long times, or at least remain in the disc for long enough to form a gas giant planet. The evolution of giant planets in circumbinary discs was considered by Nelson (2003).

In this paper, we extend the model presented in Paper I by considering the evolution of multiple planets embedded in a circumbinary disc. Here, we wish to examine how multiple planets interact with each other if they form at large distance from the binary and successively migrate toward the cavity edge. In particular, we want to look at whether or not the trapping of a planet at the cavity edge, and the subsequent migration of additional planets to its vicinity lead to growth of the planet through collisions, the formation of mean motion resonances, or destablisation of the system through gravitational scattering.

To address these issues, we first consider a system which consists of a pair of planets with masses of $m_{\mathrm{p}}=5,10$ and $20 M_{\oplus}$. We assume that one planet is trapped at the edge of the cavity while the outermost planet migrates in from larger radius. The results of the simulations show that the final outcome of such a system generally depends on the mass ratio $q=m_{\mathrm{i}} / m_{\mathrm{o}}$ (where $m_{\mathrm{i}}$ is the mass of the inner planet and $m_{\mathrm{o}}$ is the mass of the outer planet). Interestingly, we find that systems with $q \geq 1$ can reach a steady state such that the planets are locked into resonance and remain trapped at the cavity edge. Most of the systems with $q<1$, however, are unstable and lead to events such as scattering or dynamical exchange.

We performed a second set of simulations consisting of fiveplanet systems embedded in a circumbinary disc. Of three simulations performed, two resulted in a single planet orbiting around the binary because of collisions and scattering events. The remaining simulation resulted in a three-planet system remaining, with all planets in mutual mean motion resonances. This configuration was found to be stable over long time scales.

This paper is organized as follows. In Sect. 2 we describe the physical model and the numerical method. In Sect. 3 we describe the results of simulations aimed at studying the evolution of pairs of planets embedded in a circumbinary disc. We then present in Sect. 4 the simulations of five-planet systems embedded in a circumbinary disc. We finally discuss our results and present our conclusions in Sect. 4.

\section{Physical model and numerical setup}

\subsection{Disc and planet evolution}

As in Paper I, we adopt a 2D disc model for which we assume no vertical motion. The equations governing the disc evolution are described in detail in Paper I and therefore will not be discussed here.

In Paper I, the planet orbit and the disc midplane were assumed to be coplanar. However, the simulations presented here examine the evolution of multiple planets which can strongly interact with each other as their orbits converge, leading eventually to close encounters. During such an event, a planet may receive a significant component of acceleration in the vertical direction, reducing thereby the interaction with both the disc and other planets. As a consequence, we decided here to use a model in which planets can evolve in the $z$ direction as well. With respect to coplanar orbits, this will also reduce the collision rate between planets, increasing thereby the time during which planets can strongly interact.

In the work presented here, each planet can interact with every other one and with the disc. The latter interaction is expected to lead not only to orbital migration but also to eccentricity and inclination damping. The gravitational potential of the disc is calculating using the following expression:

$$
\Phi_{\mathrm{d}}=-G \int_{S} \frac{\Sigma\left(\boldsymbol{r}^{\prime}\right) \mathrm{d} \boldsymbol{r}^{\prime}}{\sqrt{r^{\prime 2}+r_{p}^{2}-2 r^{\prime} r_{p} \cos \left(\phi^{\prime}-\phi_{p}\right)+z_{p}^{2}+\epsilon^{2}}}
$$

where $\Sigma$ is the disc surface density and where the integral is performed over the disc surface. $r_{p}, \phi_{p}$ and $z_{p}$ are respectively the radial, azimuthal and vertical coordinates of the $p$ th planet. $\epsilon$ is a smoothing parameter which is set to $\epsilon=0.6 H$, where $H$ is the local disc scale height. Under the action of this gravitational potential, each planet undergoes both orbital migration and eccentricity damping. However, because of the 2D disc model we use here, bending waves cannot be launched in the disc, and so there is no disc induced damping of inclination. To model the latter we follow Tanaka \& Ward (2004) and mimic the effect of bending waves by applying to each planet a vertical force $F_{z}$ given by:

$F_{z}=\beta \frac{m_{\mathrm{p}} \Sigma_{\mathrm{p}} \Omega}{c_{\mathrm{s}}^{4}}\left(2 A_{z}^{\mathrm{c}} v_{z}+A_{z}^{\mathrm{s}} z \Omega\right)$,

where $c_{\mathrm{s}}$ is the sound speed and where $\Omega$ and $\Sigma_{\mathrm{p}}$ are respectively the Keplerian angular velocity and the disc surface density at the position of the planet. $A_{z}^{\mathrm{c}}$ and $A_{z}^{\mathrm{s}}$ are dimensionless coefficients which are set to $A_{z}^{\mathrm{c}}=-1.088$ and $A_{z}^{\mathrm{s}}=-0.871$ (Tanaka \& Ward 2004), and $\beta$ is a free parameter which is chosen such that the inclination damping $t_{\mathrm{i}}$ timescale obtained in the simulations is approximatively equal to the eccentricity damping timescale $t_{\mathrm{e}}$. Test simulations show that choosing $\beta=0.33$ give similar values for $t_{\mathrm{i}}$ and $t_{\mathrm{e}}$. We adopt therefore this value for this work. Here, it is worthwhile to notice that according to the linear theory (Tanaka \& Ward 2004), a small value of the planet inclination $\left(i_{\mathrm{p}} \ll H / R\right)$ is not expected to affect the migration rate of the planet. For large values of $i_{\mathrm{p}}$ however, migration rates may be moderately slowed down (Cresswell et al. 2007) because the interaction with the disc is reduced. Such an effect is accounted for in an approximate manner by Eq. (1).

\subsection{Numerical setup}

\subsubsection{Numerical method}

The simulations presented here were performed using the hydrodynamic code GENESIS. This code employs a second-orderaccurate method that computes advection using the monotonic transport algorithm (Van Leer 1977). Details about GENESIS are given in Paper I. All the runs use $N_{r}=256$ radial grid cells uniformly distributed between $r_{\text {in }}=0.5$ and $r_{\text {out }}=6$ and $N_{\phi}=380$ azimuthal grid cells.

The evolution of the planets plus binary system is performed using a 5th-order Runge-Kutta scheme (Press et al. 1992). In spite of the accuracy of this integrator, experiments have shown that to ensure energy conservation during close encounters, the 
time step size $\Delta t$ used to make the system evolve should be smaller than the hydrodynamical time step $\Delta t_{\mathrm{h}}$ based on the CFL criterion (Stone \& Norman 1992). Following Cresswell \& Nelson (2006), we ensure good energy conservation and accuracy by setting the time step size to $\Delta t=\min \left(\Delta t_{\mathrm{h}}, \Delta t_{n 1}, \Delta t_{n 2}\right)$, where:

$\Delta t_{n 1}=\frac{2 \pi}{400} \min _{p, p^{\prime}}\left(\sqrt{\frac{\left|\boldsymbol{r}_{p p^{\prime}}\right|^{3}}{G\left(m_{\mathrm{p}}+m_{\mathrm{p}}^{\prime}\right)}}\right)$,

and

$\Delta t_{n 1}=\frac{2 \pi}{400} \min _{p, s}\left(\sqrt{\frac{\left|\boldsymbol{r}_{p s}\right|^{3}}{G\left(m_{\mathrm{p}}+m_{s}\right)}}\right)$.

In the previous expressions, $\boldsymbol{r}_{p p^{\prime}}$ is the distance between the planets $p$ and $p^{\prime}$ and $\boldsymbol{r}_{p s}$ is the distance between the planet $p$ and star $s$. We note that throughout our simulations, the time step size is $\sim 1 / 800$ the binary orbital period.

As in Paper I, we adopt computational units in which the total mass of the binary is $M_{*}=1$, the gravitational constant is $G=1$, and the radius $r=2$ in the computational domain corresponds to $5 \mathrm{AU}$. The unit of time is $\Omega^{-1}=\sqrt{G M_{*} / a_{\mathrm{b}}^{3}}$, where $a_{\mathrm{b}}=0.4$ is the initial semimajor axis of the binary. This corresponds to an initial separation between the two stars of $\sim 1 \mathrm{AU}$.

In the simulations presented here, close encounters between two planets can result in a physical collision. Here, this is supposed to occur whenever the mutual distance $d_{p p^{\prime}}$ between planets $p$ and $p^{\prime}$ is less than $\left(3 m_{\mathrm{p}} / 4 \pi \rho\right)^{1 / 3}+\left(3 m_{\mathrm{p}}^{\prime} / 4 \pi \rho\right)^{1 / 3}$, where $\rho$ is the mass density which we assume to be the same for each planet and equal to $\rho=3 \mathrm{~g} \mathrm{~cm}^{-3}$. If a collision is found to occur between the planets $p$ and $p^{\prime}$, these are assumed to merge and are subsequently substituted by a single body with mass $m_{\mathrm{p}}+m_{\mathrm{p}}^{\prime}$. The position and velocity of the latter are set to the position and velocity of the centre of mass of the planets $p$ and $p^{\prime}$.

\subsubsection{Initial conditions}

As in Paper I, the disc aspect ratio $H / R$ is assumed to be constant and equal to $H / R=0.05$. We use also the "alpha" prescription of Shakura \& Sunyaev (1973) to model the disc anomalous kinematic viscosity $v=\alpha c_{\mathrm{s}} H$, where $c_{\mathrm{s}}$ is the isothermal sound speed and where $\alpha=10^{-4}$. The reason for choosing such a low $\alpha$ value is discussed in detail in Paper I, but is essentially because a larger value causes rapid evolution of the binary orbit that would prohibit the long simulations we present here.

In Paper I, we showed that both the disc and binary evolve toward a near-steady state as they interact with each other. From the time this equilibrium configuration is reached, the apsidal lines of the disc and binary are aligned. Also, the disc structure and the orbital elements of the binary remain essentially constant. For example, we find that the eccentricity of a binary with mass ratio $q_{\mathrm{b}}=0.1$ and initial separation $a_{\mathrm{b}}=0.4$ saturates at a value of $e_{\mathrm{b}} \sim 0.08$. The simulations presented in Paper I of one planet interacting with a circumbinary disc were performed using this quasi-equilibrium state as initial conditions for the disc and binary. Depending on the model we consider, we adopt here a similar approach when setting up our initial conditions:

i) In simulations of pairs of planets embedded in a circumbinary disc, we restart the runs presented in Paper I once the planet is trapped at the cavity edge but with a second planet evolving on a circular orbit with $a_{\mathrm{p}}=2.5$ and $i_{\mathrm{p}}=0.5^{\circ}$.
Table 1. The first column gives the run label, the second column gives the mass $m_{\mathrm{i}}$ of the inner planet and the third column gives the mass $m_{\mathrm{o}}$ of the outer planet. The fourth column gives the ratio $q=m_{\mathrm{i}} / m_{\mathrm{o}}$.

\begin{tabular}{cccc}
\hline \hline Run & $m_{\mathrm{i}}\left(M_{\oplus}\right)$ & $m_{\mathrm{o}}\left(M_{\oplus}\right)$ & $q$ \\
\hline Run1 & 10 & 10 & 1 \\
Run2 & 10 & 5 & 2 \\
Run3 & 20 & 10 & 2 \\
Run4 & 20 & 5 & 4 \\
Run5 & 5 & 10 & 0.5 \\
Run6 & 10 & 20 & 0.5 \\
Run7 & 5 & 20 & 0.25 \\
\hline
\end{tabular}

The latter is allowed to interact with the disc whose mass is $M_{\mathrm{d}} \sim 0.01 M_{\star}$ and with the other planet and binary.

ii) In simulations that evolve five-planet systems in a circumbinary disc, we embed the planets in the disc once the latter and the binary have reached a stationary state, as described in Paper I. We set the innermost planet at $a_{\mathrm{p}}=1.8$ and then calculate the initial location of the others by asssuming that two adjacent bodies $p$ and $p^{\prime}$ are separated by $\sim 5 R_{\mathrm{mH}}$, where $R_{\mathrm{mH}}$ is the mutual Hill radius defined by:

$R_{\mathrm{mH}}=\left(\frac{m_{p}+m_{p^{\prime}}}{3 M_{\star}}\right)^{1 / 3}\left(\frac{a_{p}+a_{p^{\prime}}}{2}\right)$.

Each body is assumed to initially evolve on a circular orbit with $i_{\mathrm{p}}=0.5^{\circ}$. Note that the initial separation between planets we adopt is greater that the critical value of $\sim 3.46 R_{\mathrm{mH}}$ below which rapid instability occurs for two planets on initially circular orbits (Gladman 1993).

\section{Evolution of pairs of planets embedded in circumbinary discs}

In Paper I we considered planets with masses $m_{\mathrm{p}}=5,10$ and $20 M_{\oplus}$. Therefore, we adopt here the same values for the mass $m_{\mathrm{i}}$ of the innermost planet which is assumed to be trapped at the cavity edge of the disc. For each value of $m_{\mathrm{i}}$, we have performed two or three runs for which $m_{\mathrm{o}}$ was varied between $5 \leq m_{\mathrm{o}} \leq$ $20 M_{\oplus}$. Table 1 gives the values of $m_{\mathrm{i}}, m_{\mathrm{o}}$ and $q=m_{\mathrm{i}} / m_{\mathrm{o}}$ for each run. An interesting feature of the results of these simulations is that varying the value of $q$ can lead to different outcomes. Below, we discuss in detail the different modes of evolution obtained in the simulations, and how they change depending on whether or not $q \geq 1$.

\subsection{Models with $q=1$}

The orbital evolution of a pair of planets with masses of $m_{\mathrm{p}}=$ $10 M_{\oplus}$ is shown in the left panel of Fig. 1. At the beginning of the simulation, corotation torques cause the inner planet to remain trapped at the edge of the disc cavity (see Paper I) located at $r \sim 1.2$. The outer planet, which initially evolves on a circular orbit with $a_{\mathrm{o}}=2.5$, undergoes the usual type I migration and drifts inward. As it migrates, its eccentricity $e_{\mathrm{o}}$ slowly increases due to the influence of the binary (see bottom left panel of Fig. 1). Also, the distance between the two bodies becomes smaller and smaller which can potentially leads to the formation of mean motion resonances (Papaloizou \& Szuszkiewicz 2005). Here, we find that the two planets become captured into the $4: 3$ resonance at $t \sim 1.9 \times 10^{4}$ binary orbits. The top right panel of Fig. 1 displays the evolution of both the apsidal angle $\Delta \omega=\omega_{\mathrm{i}}-\omega_{\mathrm{o}}$ and the resonant angle $\psi=4 \lambda_{\mathrm{o}}-3 \lambda_{\mathrm{i}}-\omega_{\mathrm{i}}$, where $\lambda_{\mathrm{i}}\left(\lambda_{\mathrm{o}}\right)$ and $\omega_{\mathrm{i}}\left(\omega_{\mathrm{o}}\right)$ 

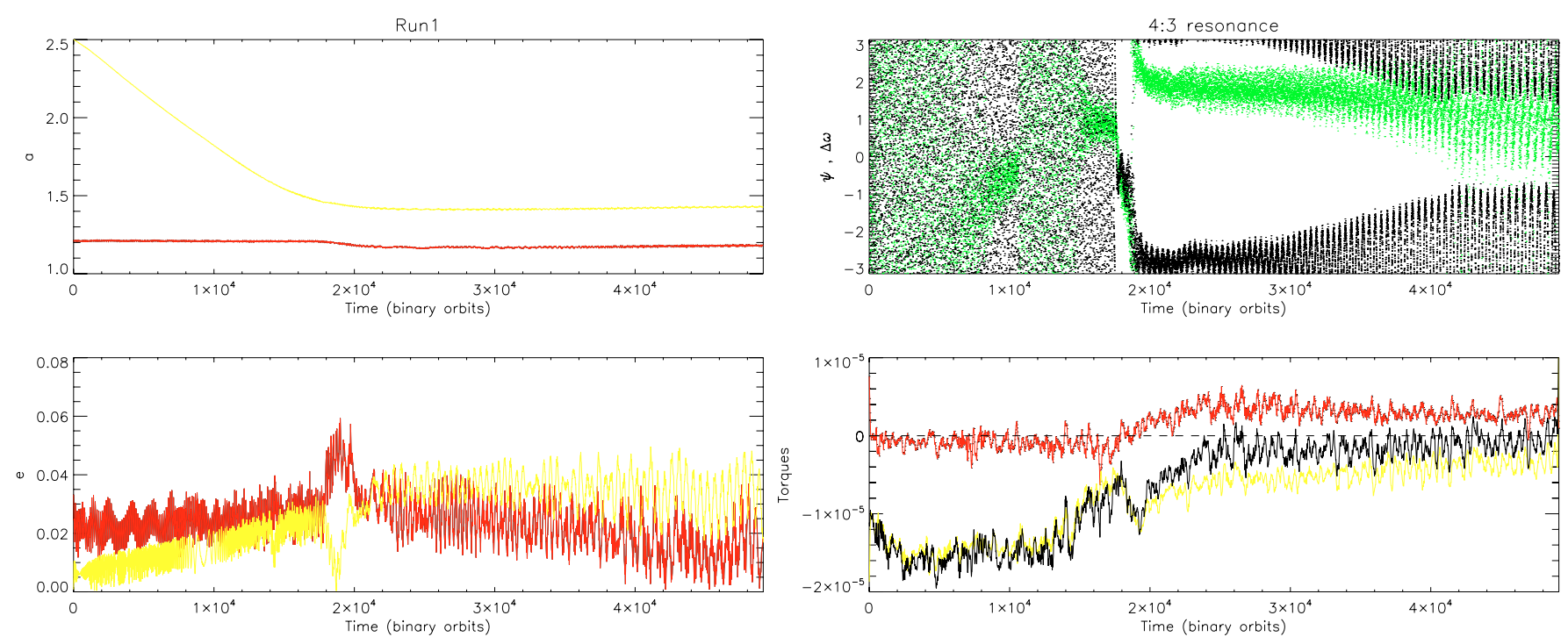

Fig. 1. Left: evolution of the semimajor axes (top panel) and eccentricities (bottom panel) of planets with masses of $m_{\mathrm{p}}=10 M_{\oplus}$. Right: the top panel shows the evolution of both the resonant angle $\psi=4 \lambda_{0}-3 \lambda_{\mathrm{i}}-\omega_{\mathrm{i}}$ associated with the 4:3 resonance (black) and the apsidal angle $\Delta \omega=\omega_{\mathrm{i}}-\omega_{\mathrm{o}}$ (green). The bottom panel shows the evolution of the torques exerted by the disc on the innermost planet (red line) and on the outermost one (yellow line). The sum (red+yellow) is displayed with the black line and corresponds to the torques exerted on the whole system.

are respectively the mean longitude and longitude of pericentre of the inner (outer) planet. Once the resonance is established, the libration amplitude of $\psi$ slightly increases with time until $t \sim 4 \times 10^{4}$ binary orbits, and then remains almost unchanged, suggesting that the planets are stably locked into the resonance. From this time onward, the system is close to an equilibrium state with both planets having constant semimajor axes and eccentricities. At the end of the simulation, the ratio of semimajor axes is $a_{\mathrm{i}} / a_{0} \sim 0.85$ and the ratio of eccentricities is $e_{\mathrm{i}} / e_{\mathrm{o}} \sim 0.6$. Such a configuration can be achieved because the torques exerted by the disc on each planet act in an opposite way and can eventually counterbalance each other. From the time the resonance is established the negative torques exerted by the disc on the outer planet make the two planets migrate inward together. However, as both planets migrate, the innermost planet experiences stronger positive corotation torques which tend to push the pair of planets outward. The bottom right panel of Fig. 1 shows the evolution of the torques exerted on each planet as well as the effective torques acting on the whole system. We see that as the evolution proceeds, the torques exerted on the inner planet are able to exactly counterbalance the ones exerted on the outer body, which leads consequently to a zero net torque acting on the system. This happens from $t \sim 2.1 \times 10^{4}$, thereby stopping the joined migration of the planets.

\subsection{Models with $q \geq 1$}

Stable resonant locking was also found in some of the calculations with $q \geq 1$. Figure 2 shows the results for Run2 in which planets have masses of $m_{\mathrm{i}}=10$ and $m_{\mathrm{o}}=5 M_{\oplus}$. With respect to Run1, the outer planet migrates more slowly since its mass is smaller. However, the mode of evolution found in Run2 is very similar to the one obtained in Run1, leading ultimately to a stable configuration with the two planets trapped in the $4: 3$ resonance from $t \sim 5 \times 10^{4}$. At earlier times, the evolution of $e_{\mathrm{o}}$ shows some peaks at $t \sim 1.9 \times 10^{4}$ and $t \sim 3 \times 10^{4}$ which coincide with the planets being temporary captured in the $2: 1$ and $3: 2$ resonances. At the end of the simulation, $e_{\mathrm{o}}$ is still slightly increasing whereas $e_{\mathrm{i}}$ is slightly decreasing, which indicates that the
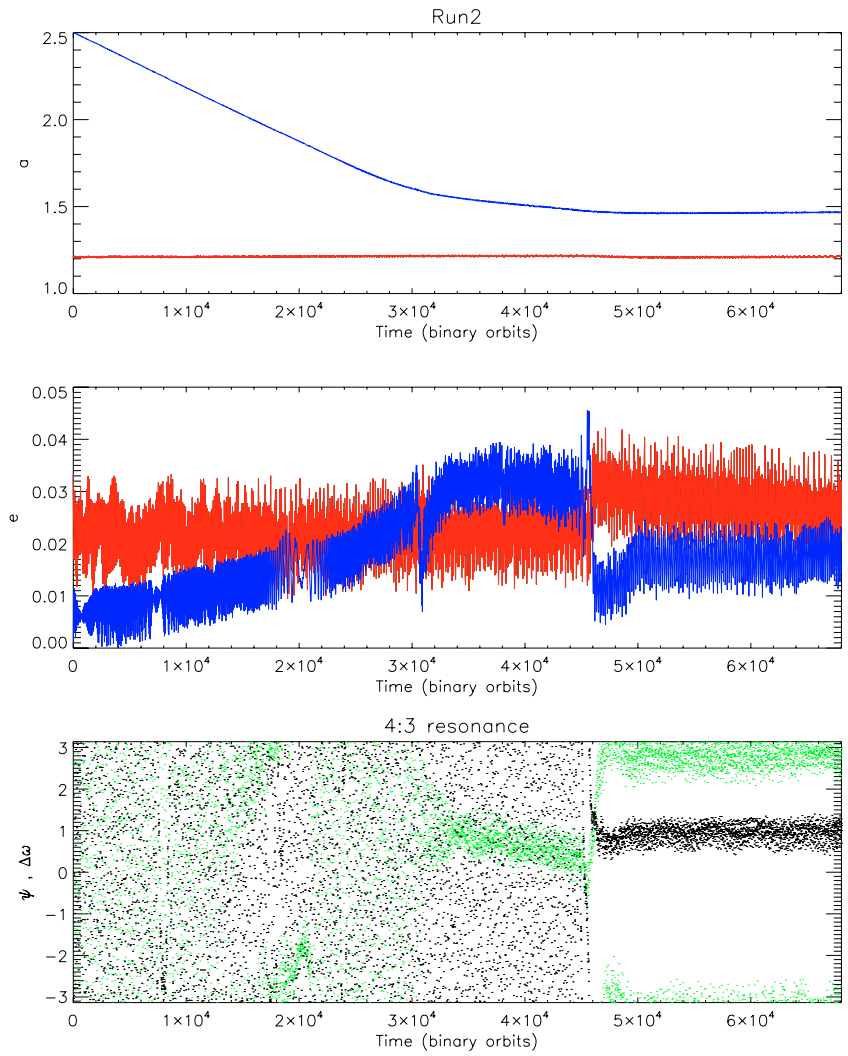

Fig. 2. This figure shows the evolution of the semimajor axes and eccentricities for Run2 in which planets have masses of $m_{\mathrm{i}}=10 M_{\oplus}$ (red line) and $m_{\mathrm{o}}=5 M_{\oplus}$ (blue line). The bottom panel displays the evolution of both the resonant angle $\psi=4 \lambda_{\mathrm{o}}-3 \lambda_{\mathrm{i}}-\omega_{\mathrm{i}}$ (black) associated with the $4: 3$ resonance and the apsidal angle $\Delta \omega=\omega_{\mathrm{i}}-\omega_{\mathrm{o}}$ (green).

equilibrium configuration is not fully established. Nonetheless, comparing Figs. 1 and 2, we can see that the libration amplitude of the resonant angle is much smaller in Run2 than in Run1, suggesting that the $4: 3$ resonance is more stable in this case. 

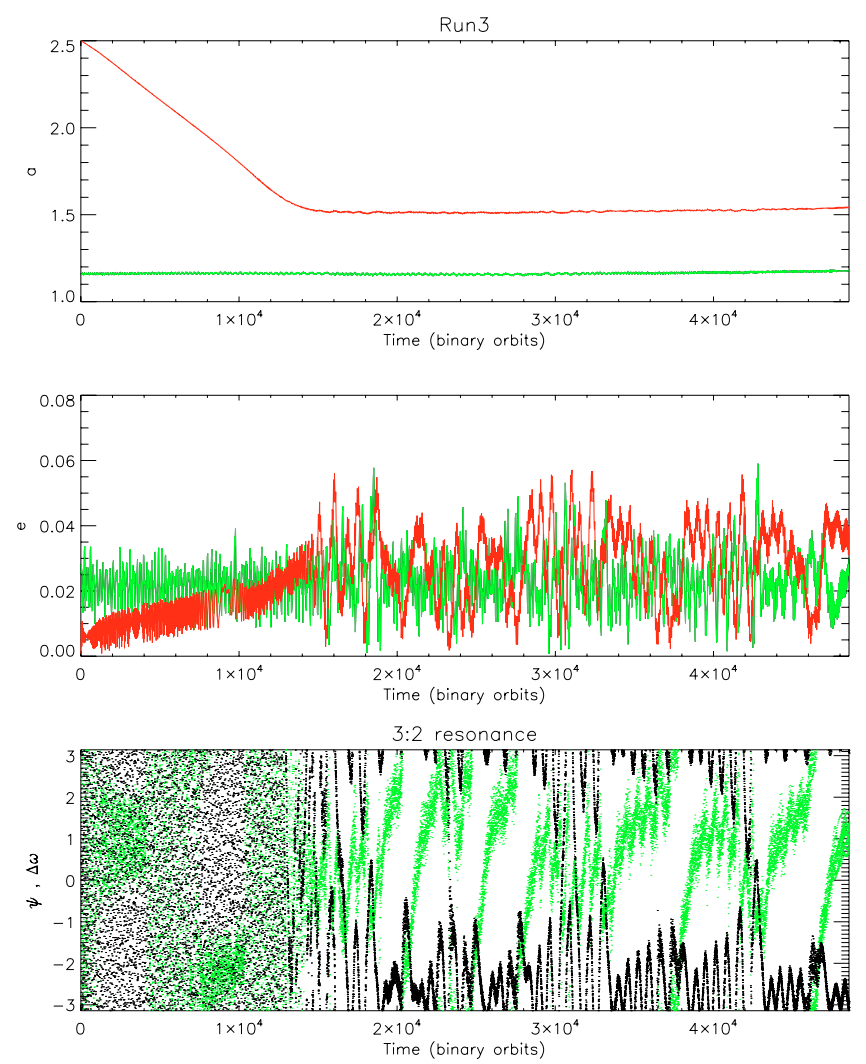

Fig. 3. This figure shows the evolution of the semimajor axes and eccentricities for Run3 in which planets have masses of $m_{\mathrm{i}}=20 M_{\oplus}$ (green line) and $m_{\mathrm{o}}=10 M_{\oplus}$ (red line). The bottom panel displays the evolution of both the resonant angle $\psi=3 \lambda_{\mathrm{o}}-2 \lambda_{\mathrm{i}}-\omega_{\mathrm{i}}$ (black) associated with the 3:2 resonance and the apsidal angle $\Delta \omega=\omega_{\mathrm{i}}-\omega_{\mathrm{o}}$ (green).

In models with $m_{\mathrm{i}}=20 M_{\oplus}$, the simulations resulted in different modes of evolution, depending on the mass of the outer planet $m_{0}$. Figures 3 and Fig. 4 display the results of calculations with $m_{\mathrm{o}}=10 M_{\oplus}$ and $m_{\mathrm{o}}=5 M_{\oplus}$ respectively. Comparing these two figures, we can see that the final state of the system is quite similar in both cases, with planets evolving on fixed orbits with $a_{\mathrm{i}} \sim 1.2$ and $a_{\mathrm{o}} \sim 1.6$. In the run with $m_{\mathrm{o}}=10 M_{\oplus}$, a 3:2 resonance forms at $t \sim 1.4 \times 10^{4}$. In the simulation with $m_{\mathrm{o}}=5 M_{\oplus}$ however, there is no evidence that the planets are in mean motion resonance, even though the system is close to the $3: 2$ commensurability. In this case, examination of the torques exerted by the disc (see upper panel of Fig. 5) reveals that the migration of the system stalls because the torques acting on both planets cancel. Here, such an effect arises because the mass of the inner planet is high enough to make the onset of non-linear effects possible. These can significantly alter the surface density profile and widen the size of the inner cavity. Indeed, we find that the edge of the inner cavity is located at $r \sim 1.2$ in simulations with $m_{\mathrm{i}}=10 M_{\oplus}$ and $m_{\mathrm{i}}=5 M_{\oplus}$ whereas the bottom panel of Fig. 5 shows that it is located at $r \sim 1.5$ in Run4. Consequently, the evolution of the system in Run4 is such that the migration of the outermost planet is halted at the edge of the cavity formed by the binary plus inner planet system, therefore preventing capture in the 3:2 resonance (or in resonances of higher degree such as $4: 3)$.
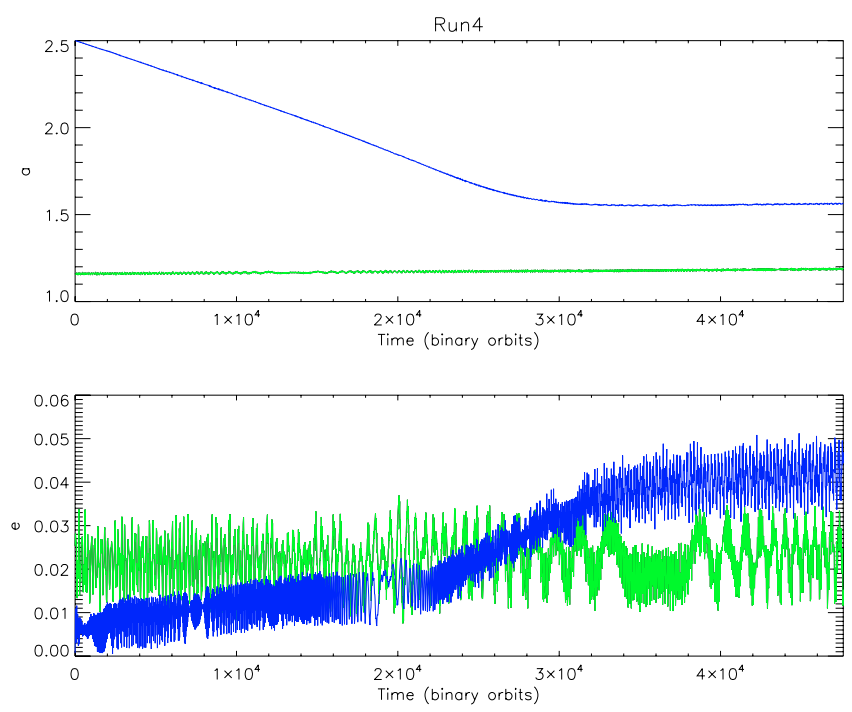

Fig. 4. This figure shows the evolution of the semimajor axes and eccentricities for Run4 in which planets have masses of $m_{\mathrm{i}}=20 M_{\oplus}$ (green line) and $m_{\mathrm{o}}=5 M_{\oplus}$ (blue line).

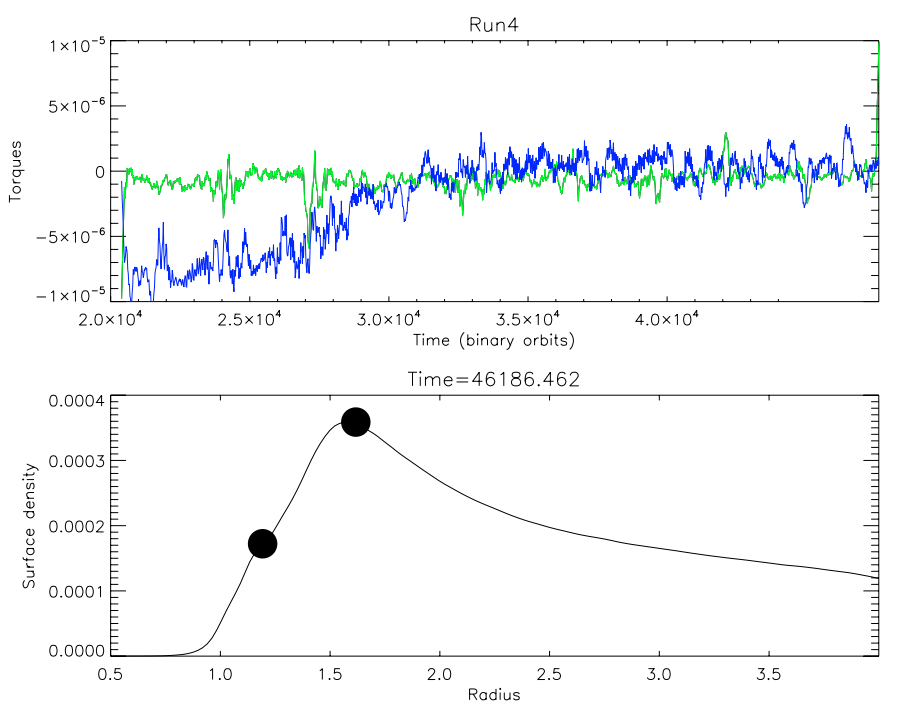

Fig. 5. The upper panel shows the evolution of the torques exerted by the disc on the planets for Run4. The green line corresponds to the torques exerted on the $20 M_{\oplus}$ planet and the blue line corresponds to the torques exerted on the $5 M_{\oplus}$ planet. The lower panel displays the azimutal average of the surface density at the time shown above the plot as well as the position of the planets at the same time.

\subsection{Models with $q<1$}

For simulations with $q<1$, the evolution of the system differed significantly from that just described in all but one case (Run6). Figure 6 shows the results of a simulation (Run5) with $q=0.5$ in which planets have masses of $m_{\mathrm{i}}=5 M_{\oplus}$ and $m_{\mathrm{o}}=10 M_{\oplus}$. Here, the outer planet passes through the $4: 3$ resonance at $t \sim 1.8 \times 10^{4}$ and then slips into the $6: 5$ resonance with the inner planet at $t \sim 2 \times 10^{4}$. The passage through these resonances is clearly accompanied by an increase of the inner planet eccentricity $e_{\mathrm{i}}$. At $t \sim 2.1 \times 10^{4}$, the inner planet undergoes a close encounter with the outer one as a result of resonant trapping. This subsequently leads to the scattering of the inner planet further out in the disc while the outer one is pushed inward by virtue of conservation of angular momentum. Interestingly, such an orbital 

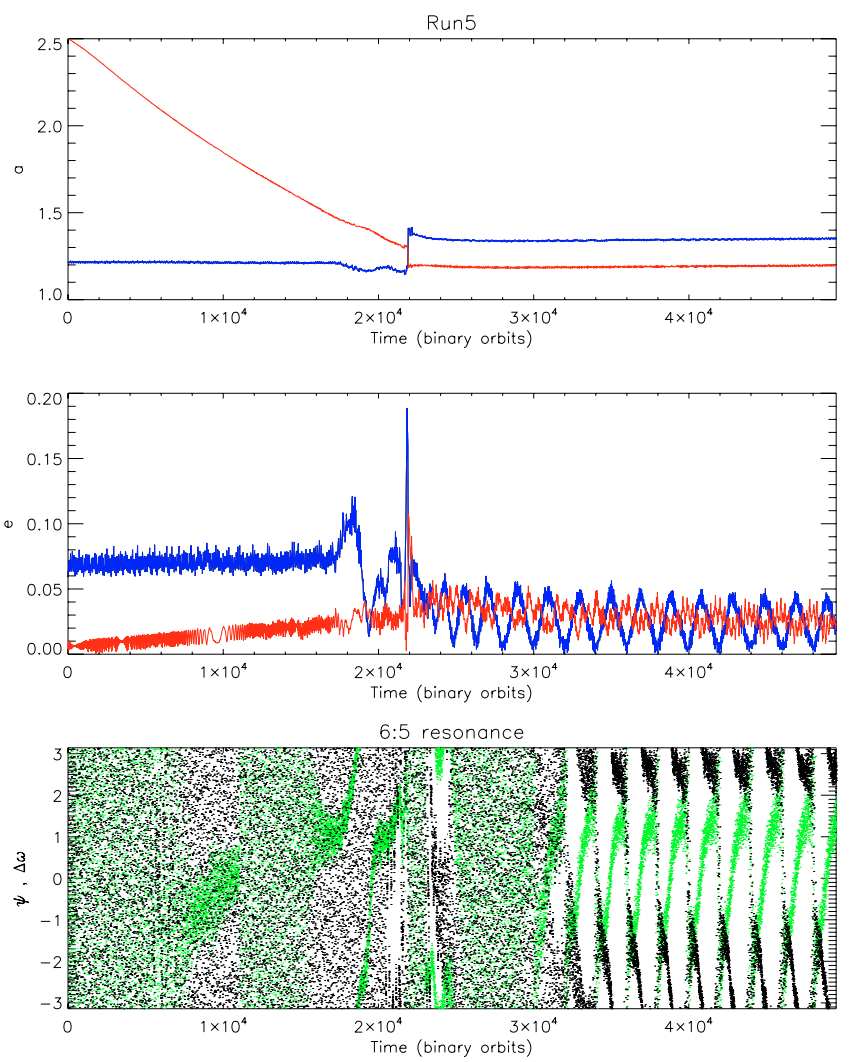

Fig. 6. This figure shows the evolution of the semimajor axes and eccentricities for Run5 in which planets have masses of $m_{\mathrm{i}}=5 M_{\oplus}$ (blue line) and $m_{\mathrm{o}}=10 M_{\oplus}$ (red line). The bottom panel displays the evolution of both the resonant angle $\psi=6 \lambda_{\mathrm{o}}-5 \lambda_{\mathrm{i}}-\omega_{\mathrm{i}}$ (black) associated with the $6: 5$ resonance and the apsidal angle $\Delta \omega=\omega_{\mathrm{i}}-\omega_{\mathrm{o}}$ (green).

exchange leads to a configuration of the system similar to that of models with $q \geq 1$. In good agreement with what we described in Sect. 3.2, we find that the final state of the system is indeed an equilibrium configuration with the two planets locked into the 6:5 resonance. At the end of the simulation, the $5 M_{\oplus}$ and $10 M_{\oplus}$ planets are respectively located at $a_{\mathrm{i}} \sim 1.35$ and $a_{\mathrm{o}} \sim 1.2$.

Although $q$ has the same value in Run6 as it is in Run5, we find a different mode of evolution as shown in Fig. 7. In Run6 the planets have masses $m_{\mathrm{i}}=10 M_{\oplus}$ and $m_{\mathrm{o}}=20 M_{\oplus}$. Relative to Run5 the disc induced eccentricity damping acting on the innermost planet is stronger, thereby preventing eccentricities from reaching large values, and consequently preventing planets from undergoing close encounters. Figure 7 shows that the mode of evolution of the system in Run6 is almost similar to that of models with $q \geq 1$, since the planets are in resonance (the 6:5 resonance in this case) and do not migrate. At the end of the evolution, the innermost and outermost planets are respectively located at $a_{\mathrm{i}} \sim 1.1$ and $a_{\mathrm{o}} \sim 1.3$ and the ratio of eccentricities is $e_{\mathrm{i}} / e_{\mathrm{o}} \sim 1.3$.

Figure 8 shows the results of a calculation (Run7) with $m_{\mathrm{i}}=$ $5 M_{\oplus}$ and $m_{\mathrm{o}}=20 M_{\oplus}$, corresponding to a model with $q=0.25$. Here, the $20 M_{\oplus}$ planet enters the 4:3 resonance with the $5 M_{\oplus}$ body at $t \sim 9 \times 10^{3}$, which drives the eccentricity of the latter upward. This occurs until the inner planet has a close encounter with the binary, resulting in the planet being completely ejected from the system. At later times, the evolution is close to that described in Paper I, with the $20 M_{\oplus}$ planet migrating until it is trapped at the edge of the cavity formed by the binary.
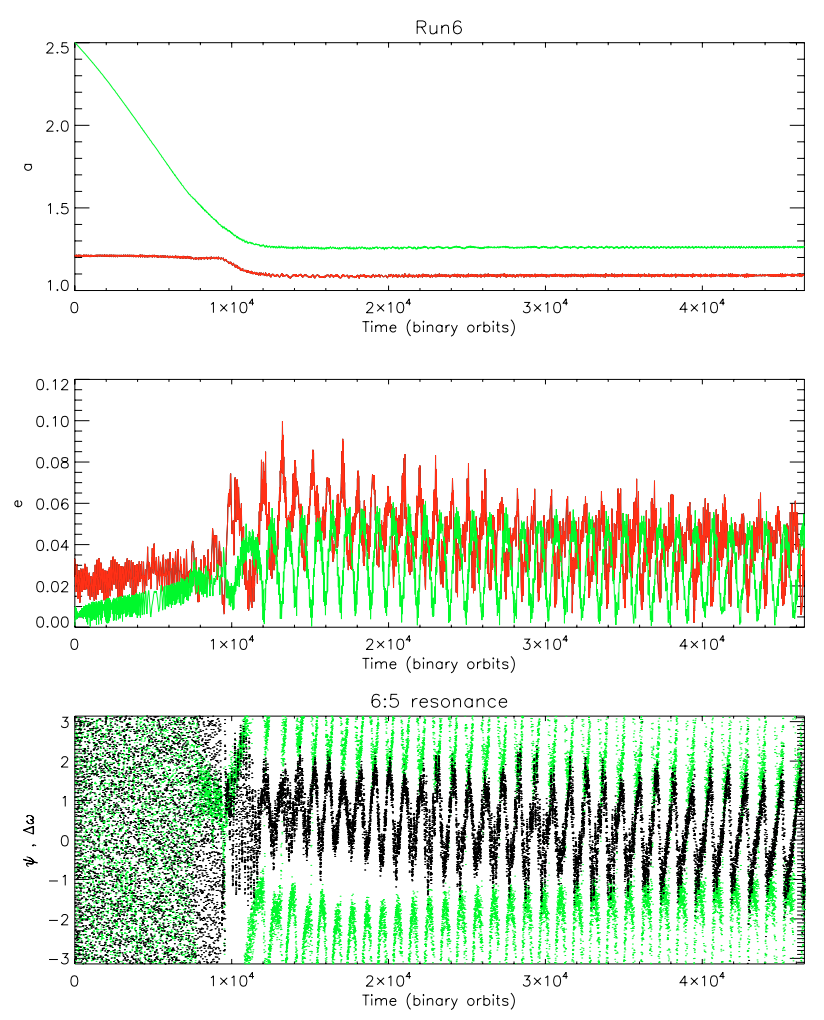

Fig. 7. This figure shows the evolution of the semimajor axes and eccentricities for Run6 in which planets have masses of $m_{\mathrm{i}}=10 M_{\oplus}$ (red line) and $m_{\mathrm{o}}=20 M_{\oplus}$ (green line). The bottom panel displays the evolution of both the resonant angle $\psi=6 \lambda_{\mathrm{o}}-5 \lambda_{\mathrm{i}}-\omega_{\mathrm{i}}$ (black) associated with the 6:5 resonance and the apsidal angle $\Delta \omega=\omega_{\mathrm{i}}-\omega_{\mathrm{o}}$ (green).
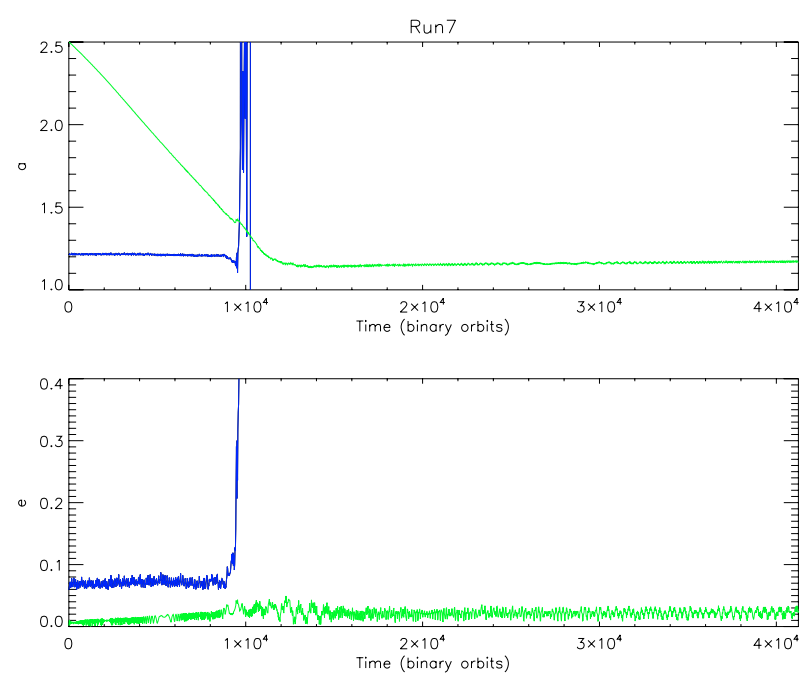

Fig. 8. This figure shows the evolution of the semimajor axes and eccentricities for Run6 in which planets have masses of $m_{\mathrm{i}}=5 M_{\oplus}$ (blue line) and $m_{\mathrm{o}}=20 M_{\oplus}$ (green line).

\section{Evolution of five-planet systems embedded in circumbinary discs}

We now turn to the question of how a swarm of planets embedded in a circumbinary disc evolves. To address this issue, we have considered different models in which five planets having masses of $5,7.5,10,12.5$ and $15 M_{\oplus}$ interact with each other. Three simulations have been performed in which we 
varied the initial configuration of the system. In one run (hereafter Model1), the initial mass distribution of planets decreases as one moves out in the disc, whereas it is chosen to be random in Model2. In Model3 the initial mass distribution increases as a function of increasing orbital radius.

\subsection{Model1}

In all the simulations we have performed, the innermost planet initially evolves on a circular orbit with $a_{1}=1.8$. For this model the initial planetary mass distribution decreases outward, so that the innermost planet has mass of $m_{1}=15 M_{\oplus}$. As noted already, the planets are spaced in orbital radius assuming a mutual separation of $5 R_{\mathrm{mH}}$. Thus the other planets with masses of $m_{\mathrm{p}}=12.5,10,7.5,5 M_{\oplus}$ are located initially at $a_{\mathrm{p}}=2.1,2.4$, $2.8,3.1$ respectively.

Figure 9 shows the evolution of the semimajor axes and eccentricities of planets for this model. At the beginning of the simulation, all the planets migrate inward as a consequence of type I migration, with a migration rate decreasing as one moves from the innermost planet to the outermost one. At $t \sim 7 \times 10^{3}$, the innermost and most rapidly migrating $15 M_{\oplus}$ planet reaches the edge of the inner cavity located at $r \sim 1.2$. As expected, this body remains trapped at this location until the inwardly migrating $12.5 M_{\oplus}$ planet approaches and they enter the 4:3 resonance at $t \sim 1.1 \times 10^{4}$. From this time the evolution of these two planets is similar to that of pairs of planets with $q \geq 1$ described in Sect. 3.2: the planets reach a quasi equilibrium state such that they evolve on non migrating orbits with $a_{1} \sim 1.2$ and $a_{2} \sim 1.4$, and with eccentricities remaining almost constant. This lasts until the $10 M_{\oplus}$ planet enters the 4:3 resonance with the second body at $t \sim 1.5 \times 10^{4}$. Again, the third planet tends to push the innermost planets inward, but the corotation torques exerted on the $15 M_{\oplus}$ planet are able to counterbalance this effect and the migration of this three-planet system is stalled. A similar process occurs each time a migrating planet is resonantly captured by the bodies located inside its orbit. Over time we find that some planets slip from one resonance to another, but the system remains globally stable during these episodes. For example, the fifth planet with mass $m_{5}=5 M_{\oplus}$ slips from the 4:3 resonance with the $7.5 M_{\oplus}$ planet to the 5:4 resonance at $t \sim 4.5 \times 10^{4}$ and finally enters the $6: 5$ resonance at $t \sim 5.2 \times 10^{4}$. The final outcome of the simulation is a system forming a series of resonances between adjacent bodies with each of them evolving on a non migrating orbit.

Figure 10 displays the resonant angles $\psi_{1}=(p+1) \lambda_{\mathrm{o}}-$ $p \lambda_{\mathrm{i}}-\omega_{\mathrm{i}}$ and $\psi_{2}=(p+1) \lambda_{\mathrm{o}}-p \lambda_{\mathrm{i}}-\omega_{\mathrm{o}}$ corresponding to the $(p+1): p$ commensurabilities that form between each pair of adjacent bodies. We see that all commensurabilities that form are first order resonances, in agreement with results obtained by Cresswell \& Nelson (2006). This simulation suggests that in a circumbinary disc, corotation torques exerted at the edge of the inner cavity provide an efficient mechanism against type I migration for a swarm of planets, and that resonant capture prevents close encounters, scattering and collisions when the initial planetary mass distribution decreases as a function of orbital radius.

\subsection{Model2}

In this model, the initial configuration of the system is such that moving from the innermost planet to the outermost one, planets have masses of $m_{\mathrm{p}}=15,7.5,12.5,5$ and $10 M_{\oplus}$ respectively.

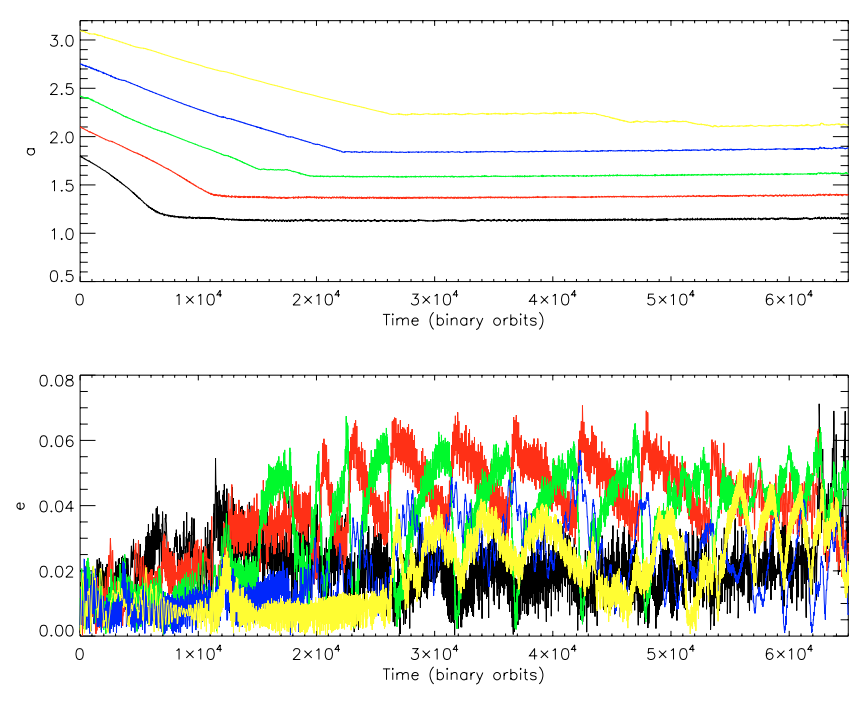

Fig. 9. This figure shows the evolution of the semimajor axes and eccentricities of planets for Model1. Moving from the innermost planet to the outermost one, planets have masses of 15 (black line), 12.5 (red line), 10 (green line), 7.5 (blue line) and $5 M_{\oplus}$ (yellow line).

A snapshost of the disc surface density at the beginning of the simulation is presented in the left panel of Fig. 12 and the evolution of the semimajor axes and eccentricities of planets for this model is illustrated in Fig. 11. Once again, the innermost $15 M_{\oplus}$ planet rapidly drifts toward the edge of the inner cavity where it remains trapped. In comparison with Model1, adjacent bodies have significantly different masses which leads to a stronger diffential migration between them. This causes the $12.5 M_{\oplus}$ and $10 M_{\oplus}$ planets to rapidly catch up with the $7.5 M_{\oplus}$ and $5 M_{\oplus}$ planets respectively, leading to the formation of resonances. Here, the $12.5 M_{\oplus}$ planet first enters the 6:5 resonance with the $7.5 M_{\oplus}$ body and then slips into the 7:6 resonance. Fig. 11 shows that the excitation of eccentricities due to this resonant interaction, and the influence of surrounding planets, leads ultimately to a collision between these two bodies at $t \sim 1.7 \times 10^{4}$. This merger forms a $20 M_{\oplus}$ planet, which then migrates inward until it becomes locked stably into the 5:4 resonance with the $15 M_{\oplus}$ planet. At $t \sim 3 \times 10^{4}$ a new collision occurs between the 5 and $10 M_{\oplus}$ planets. Earlier, these two planets passed through a sequence of different resonances and were in the 7:6 resonance just before this collision occured. Again, this newly formed $15 M_{\oplus}$ planet migrates until it catches with the $20 M_{\oplus}$ body.

The right panel of Fig. 12 shows a snapshot of the disc surface density at the end of the simulation. We see that the final state of the system consists of only three bodies evolving in the disc. Moving from the innermost planet to the outermost one, these have masses of 15,20 and $15 M_{\oplus}$. Once again, the corotation torques exerted at the cavity edge prevent inward migration, leaving each surviving body in resonance with its neighbours. Figure 13 displays the resonant angles corresponding to the commensurabilities that form between each pair. These are the 5:4 resonance for the first pair and the 4:3 resonance for the second one. This is in good agreement with the simulations performed by Cresswell \& Nelson (2006) who studied the evolution of a swarm of planets embedded in a protoplanetary disc, and who found that the 4:3, 5:4, 6:5 and 7:6 resonances are most favoured. 

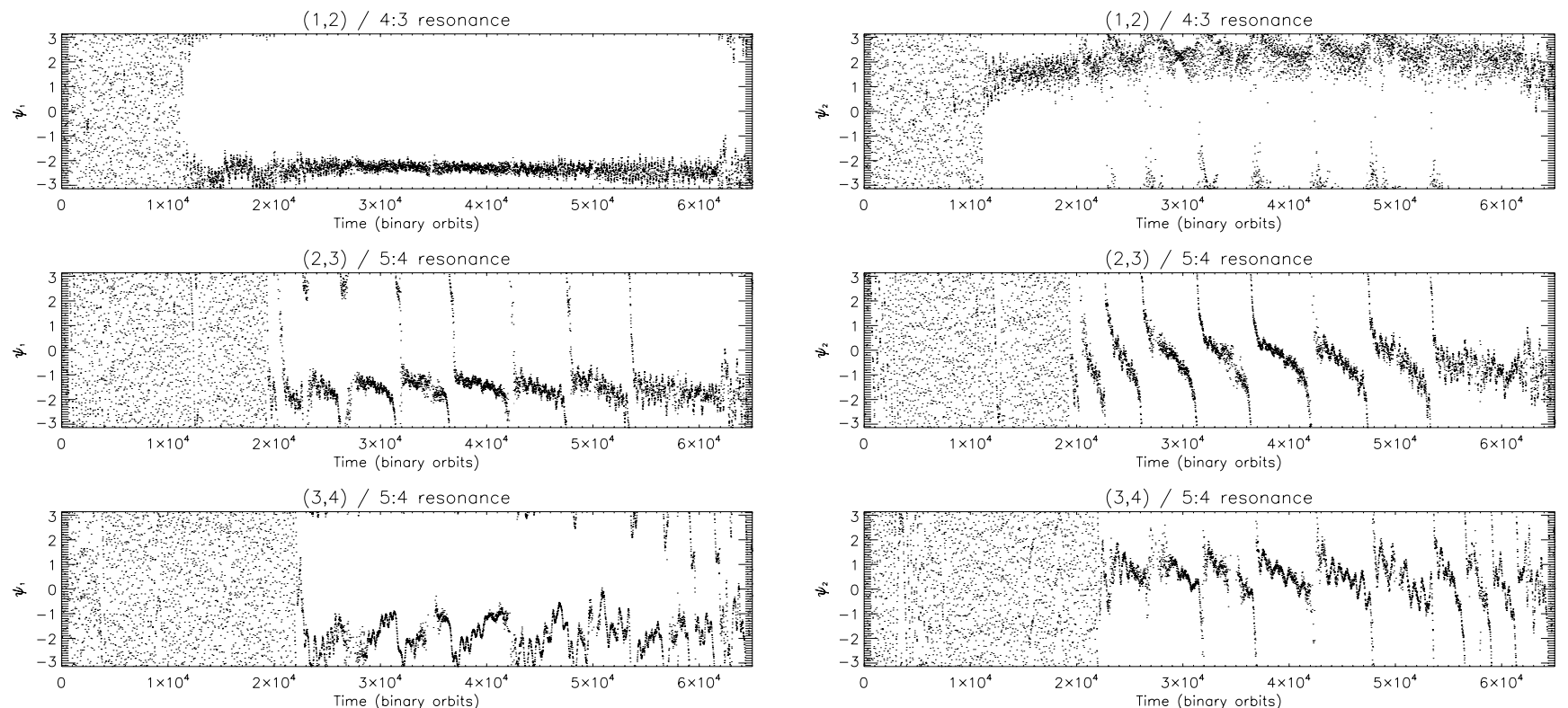

$(4,5) / 6: 5$ resonance
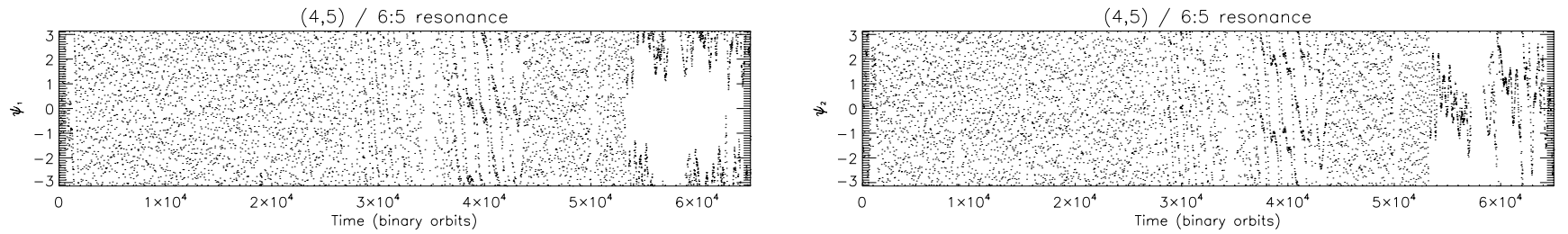

Fig. 10. This figure shows the resonances which are established between adjacent bodies at the end of the simulation corresponding to Model1. Planets are labelled from 1 to 5, with 1 being the innermost planet and 5 being the outermost one.
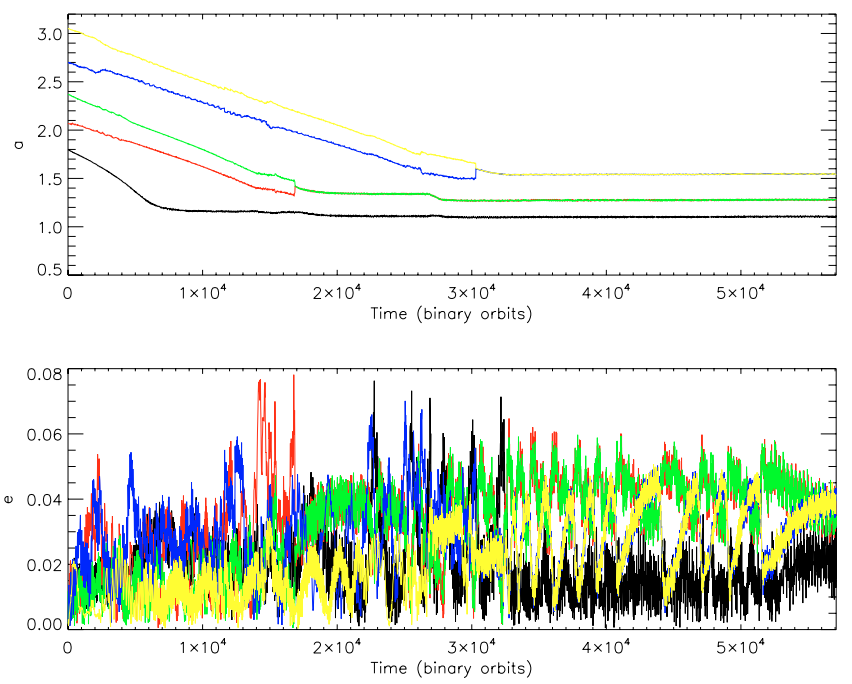

Fig. 11. This figure shows the evolution of the semimajor axes and eccentricities of planets for Model2. Moving from the innermost planet to the outermost one, planets have masses of 15 (black line), 7.5 (red line), 12.5 (green line), 5 (blue line) and $10 M_{\oplus}$ (yellow line).

\subsection{Model3}

In this last model, we consider planets with masses $m_{\mathrm{p}}=5,7.5$, $10,12.5$ and $15 M_{\oplus}$ located initially at $a_{\mathrm{p}}=1.8,2,2.3,2.7$ and 3.1 respectively.

The evolution of the semimajor axes and eccentricities of planets for Model3 are shown in Fig. 14. At the beginning of the simulation, each planet migrates inward, but the initial configuration of this model is such that the orbits of two adjacent bodies rapidly converge, leading to the formation of resonances. At $t \sim 3 \times 10^{3}$, the system has already evolved to a state where each body is in resonance with its closest neighbours, which occurs here before the innermost planet reaches the inner cavity. Moving from the inner planet to the outer one, the commensurabilities that form are respectively the 7:6, 6:5, 6:5 and 5:4 resonances. The resonant interaction between the 5 and 7.5 $M_{\oplus}$ planets increases their eccentricities up to $e_{\mathrm{p}} \sim 0.08$. Eccentricity growth within the swarm leads to crossing orbits, and subsequent interactions cause the breaking of resonances. This leads to a collison between the 5 and $7.5 M_{\oplus}$ planets at $t \sim 1.8 \times 10^{4}$. At $t \sim 2.4 \times 10^{4}$ a collision resulting in a $22.5 M_{\oplus}$ planet also occurs between the 10 and $12.5 M_{\oplus}$ bodies which were locked into the 7:6 resonance prior to this event.

The final state of the system consists of three planets with masses of $m_{\mathrm{p}}=12.5,22.5$ and $15 M_{\oplus}$ respectively located at $a_{\mathrm{p}}=1.1,1.3$ and 1.5. The upper panel of Fig. 15 shows that the 5:4 resonance is clearly established between the first pair of planets. At the end of the simulation, these two bodies evolve on non migrating orbits, whereas the outermost body migrates outward very slightly, indicating that the latter is not in resonance with its interior neighbour. The disc surface density profile at $t \sim 4 \times 10^{4}$ is displayed in the bottom panel of Fig. 15. It shows that the outermost planet is located in a region where the disc has a large positive surface density gradient, close to the outer edge of the partial gap formed by the $22.5 M_{\oplus}$ body. This results in large positive corotation torques, leading to the observed outward migration of the outermost planet. This process is likely to operate until the planet reaches a fixed point located near the gap edge, where the total torque (corotation plus Lindblad) cancels (Masset et al. 2006). This result is consistent with that found in 

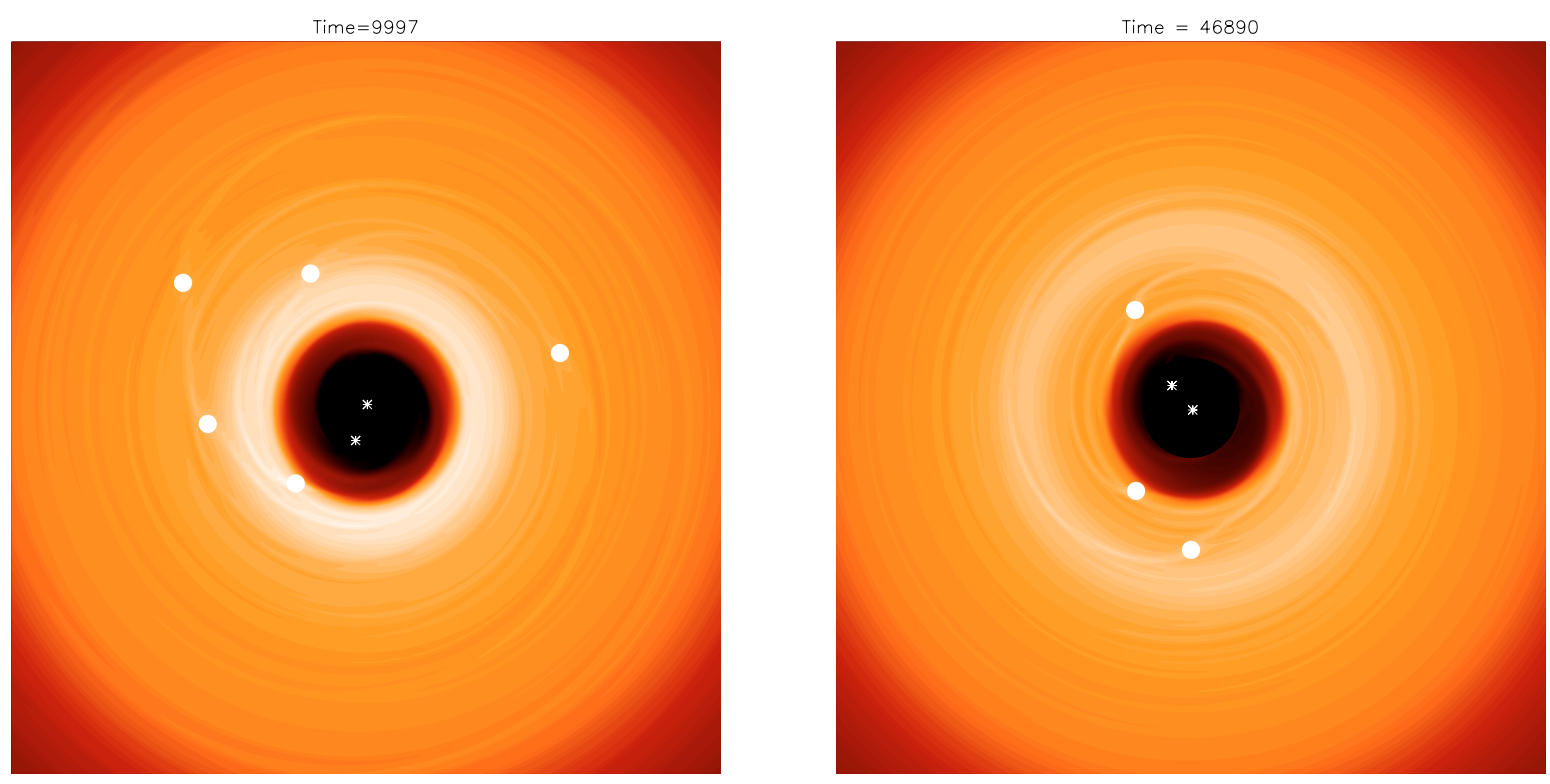

Fig. 12. This figure shows, for Model2, snapshots of the disc surface density at times shown above the plots. In this figure, planets are represented by white circles.
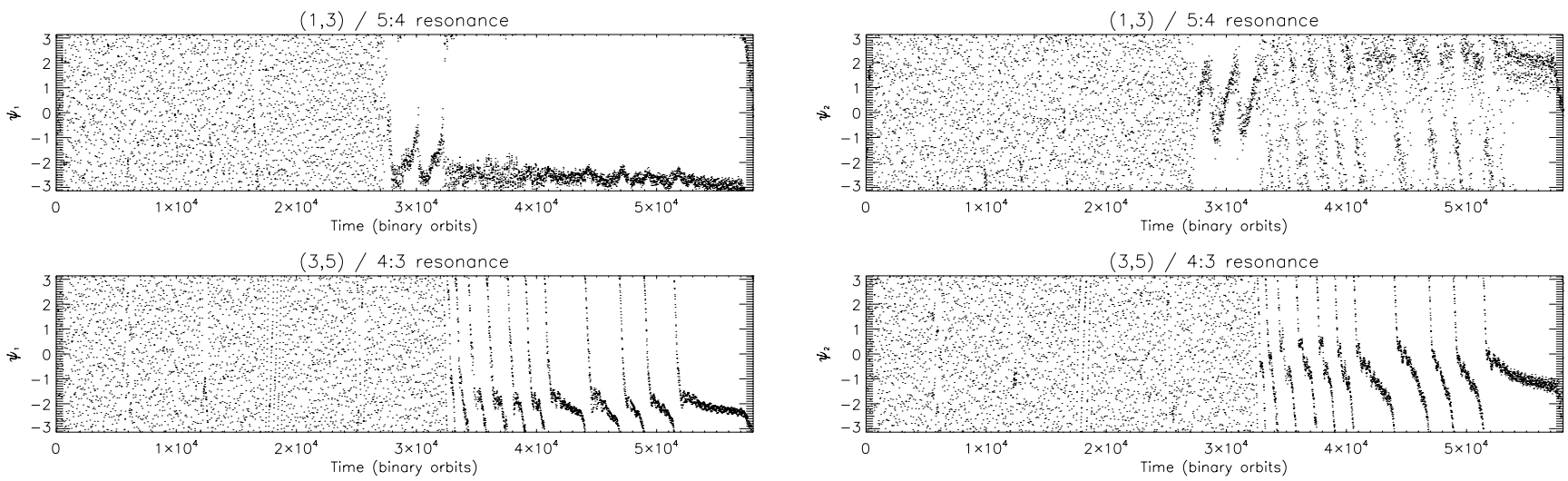

Fig. 13. This figure shows the resonances which are established between adjacent bodies at the end of the simulation corresponding to Model2. Planets are labelled from 1 to 5 , with 1 being the innermost planet and 5 being the outermost one.

Sect. 3.2 where the inner and outer planet masses were 20 and $5 M_{\oplus}$, respectively.

\subsection{Long term evolution}

We now turn to the question of the long-term evolution of the planetary systems obtained in the five-planet simulations. Because the interaction with the gas disc tends to damp eccentricities, it is necessary to examine the dynamical stability of the planets after the disc dispersal to establish long term stability. Each of the previous simulations was restarted at a point corresponding to the end of the run, but with the gas surface density decaying exponentially with an e-folding time $t_{\mathrm{dec}}=2 \times 10^{3}$. Once these systems had evolved for $\sim 10^{4}$ binary orbits, by which time the surface density in the discs had decreased by a factor of $\sim 10^{3}$, we continued the simulations with a pure $N$-body code, ignoring any residual effects of the remaining gas. For each of the five-planet models the results of this procedure are presented in Fig. 9, which displays the time evolution of the orbital radii of the planets.
In Model1, the eccentricity growth resulting from the disc dispersal gives rise, at the beginning of the simulation, to numerous scattering events that eventually lead to collisions. At time $t \sim 8 \times 10^{4}$ a system of two planets with masses of 27.5 and $22.5 M_{\oplus}$ remains, but these merge at $t \sim 1.1 \times 10^{5}$. The final state of the system is then a $50 M_{\oplus}$ planet orbiting at $r \sim 1.5$.

A similar outcome is obtained in Model3 in which the longterm evolution resulted in a $15 M_{\oplus}$ planet evolving in a higheccentricity orbit with a semi-major axis of $a_{\mathrm{p}} \sim 2$. At earlier times, the increase in eccentricities following disc dispersal led to a collision between the 12.5 and $22.5 M_{\oplus}$ bodies, thereby forming a new $35 M_{\oplus}$ planet. At $t \sim 2 \times 10^{5}$, the latter is observed to undergo a close encounter with the cental binary, leading to this body being completely ejected from the system.

Interestingly, the three-planet system in Model2 appears to be dynamically stable over long time scales, with the planets maintaining their commensurabilities. This indicates that multiplanet resonant systems could potentially be found in circumbinary discs, where the existence of the resonance helps to maintain the stability of the system. 

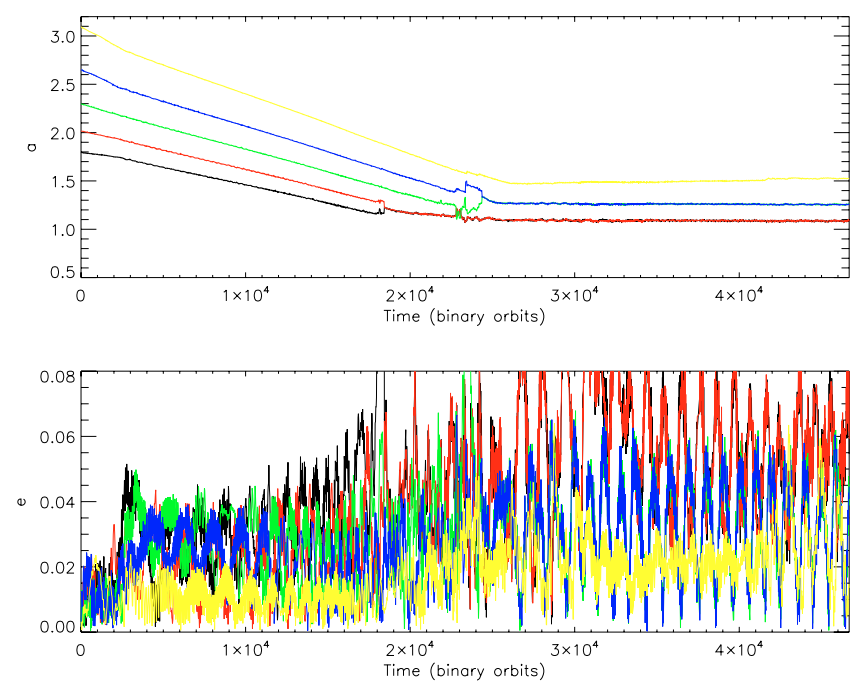

Fig. 14. This figure shows the evolution of the semimajor axes and eccentricities of planets for Model3. Moving from the innermost planet to the outermost one, planets have masses of 5 (black line), 7.5 (red line), 10 (green line), 12.5 (blue line) and $15 M_{\oplus}$ (yellow line).
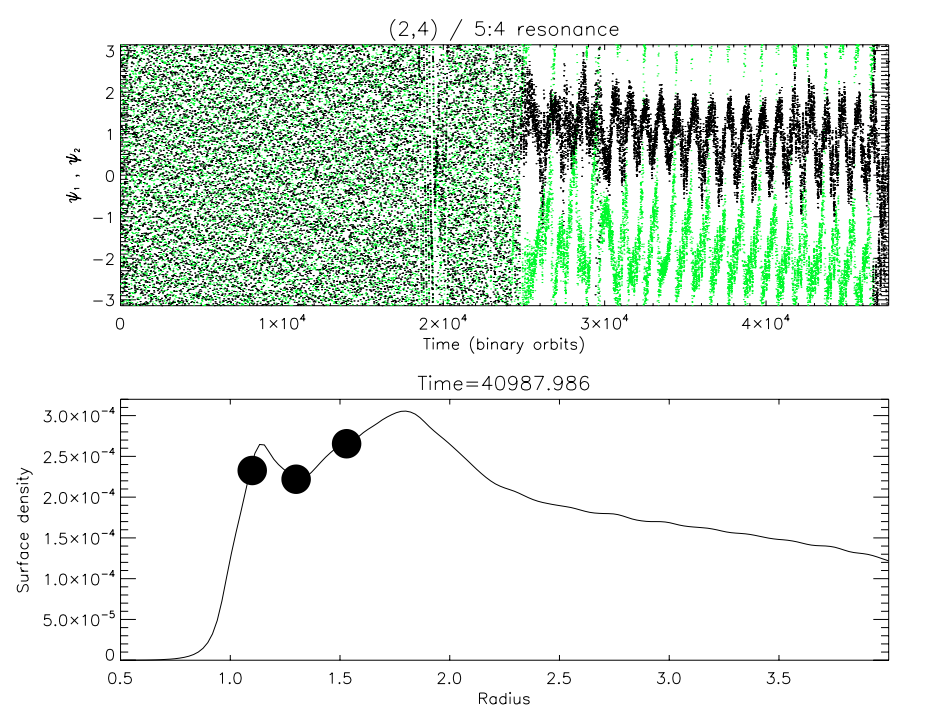

Fig. 15. The upper panel shows, for Model3, the resonant angles $\psi_{1}$ (black) and $\psi_{2}$ (green) associated with the resonance that forms between the two innermost planets. The bottom panel displays the disc surface density profile at the time shown above the plot. The semimajor axes of the planets at that time are represented by black circles.

\section{Summary and conclusion}

In this paper we have presented the results of hydrodynamic simulations aimed at studying the evolution of multiple planets embedded in a circumbinary disc.

We first focused on a system consisting of a pair of planets interacting with each other. We assumed that one body is trapped at the edge of the inner cavity formed by the binary, while the other migrates inward from outside the orbit of the innermost body. Our calculations show different outcomes, depending on the planet mass ratio $q=m_{\mathrm{i}} / m_{\mathrm{o}}$.

i) For models with $q=1$, the simulations indicate that the system evolves toward a quasi equilibrium state with both planets trapped in a mean motion resonance and evolving on non
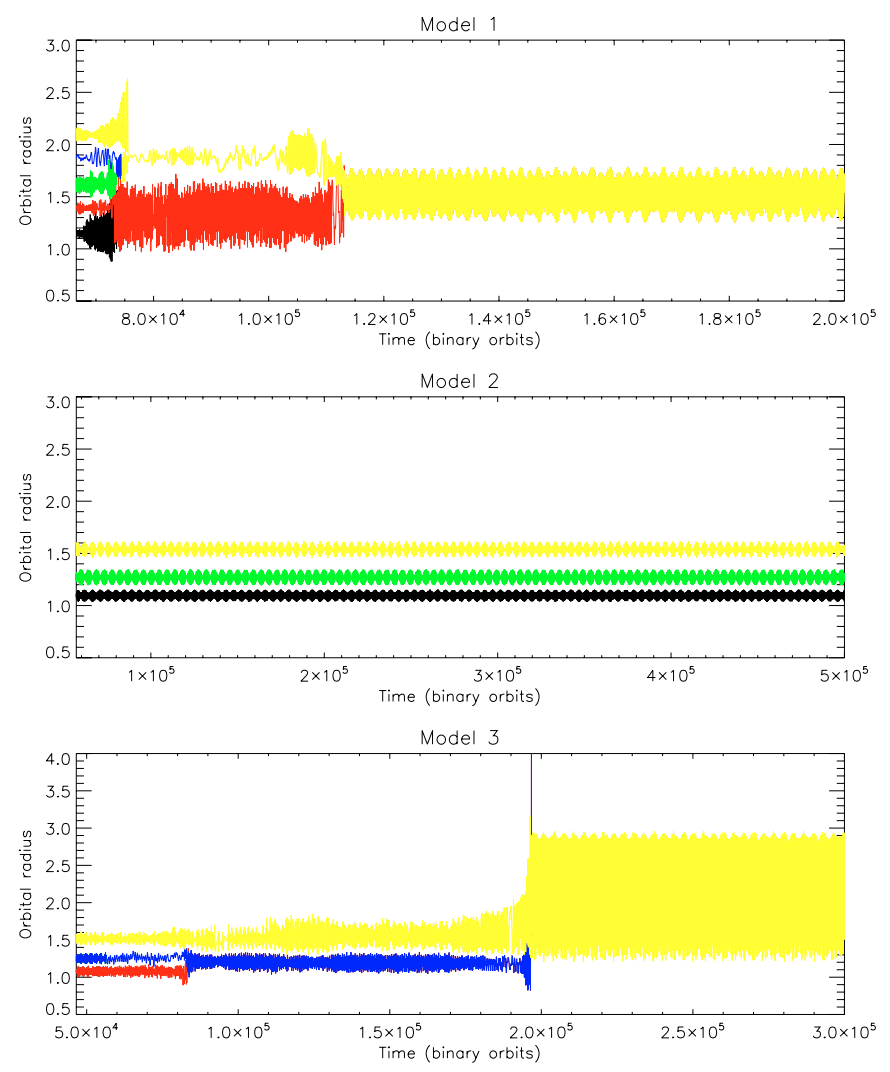

Fig. 16. This figure shows the time evolution of the orbital radii of the planets for Model1, Model2 and Model3 as a consequence of the disc dispersal. It corresponds to the results of hydro plus $N$-body simulations (see text for details).

migrating orbits. This occurs because the positive corotation torques exerted on the innermost planet counterbalance the negative corotation torques exerted on the outermost one.

ii) For models with $q \geq 1$, most of the runs resulted in a similar mode of evolution. Occasionally however, the final fate of the sytem was such that the two bodies are in close vicinity to one another, have stopped migrating, but are not in resonance. This arises when the mass of the innermost planet is high enough to open a partial gap in the disc, such that the migration of the outer planet is stopped at the edge of the large cavity formed by both the binary and the innermost planet.

iii) For most of the models with $q<1$, the planets involved in the simulations underwent different dynamical processes such as scattering or orbital exchange. When orbital exchange occurs, the final state of the system is a stable mean motion resonance with the more massive planet now being the innermost one. Scattering and orbital exchange occurs once a first order resonance is established between the planets. This drives up the eccentricity of the inner planet leading to a close encounter with either the outer planet or the binary. A close encounter with the binary leads to ejection.

Having examined the two-planet problem, we then focused on more "realistic" systems composed of five planets with masses of $5,7.5,10,12.5$ and $15 M_{\oplus}$ embedded in a circumbinary disc. We performed three simulations, with the planets being placed in different initial orbital configurations.

In general terms, the evolution of such a system proceeds as follows. Each planet migrates inward until it is captured into 
resonance with an adjacent body. This occurs either because the innermost planet has reached the edge of the inner cavity, where it remains trapped, or because two adjacent bodies migrate differentially. In some simulations, resonant interaction and the associated eccentricity growth resulted in close encounters and collisions between bodies, resulting in the formation of a more massive planet. At later times, once the more chaotic phase of evolution is over, the system behaves more quiescently, and generally reaches a stationary state with each remaining body in resonance with its closest neighbours on non migrating orbits due to planet trapping at the cavity edge. In two of the three simulations, we found that disc dispersal led to strong scattering and collisions between bodies, resulting in eventually only one planet being left in the system. In the remaining case, we found that three planets could exist stably in a three body mean motion resonance over very long times.

The results of our simulations indicate that resonant systems of planets with masses in the $\sim$ few-Earth mass range may be common in close binary systems. Indeed, the inner edge of a circumbinary disc plays the role of a barrier, which raises the possibility that two bodies become resonantly trapped. This resonance trapping, however, usually prevents the two planets from approaching each other so that they are unable to merge to form a larger body. When more than two bodies are introduced we found that the resulting scattering and close encounters could lead to planetary growth, however, but we have been unable to run sufficient numbers of simulations to provide reliable statistical information about the range of outcomes.

A number of outstanding issues remain when considering planet formation in circumbinary discs. The first is understanding where the planetary cores can form due to planetesimal accretion. The formation of an eccentric disc around a binary system, and the presence of the binary itself, can increase the velocity dispersion to a value where accretion does not occur except far out in the disc. The second is understanding the growth of cores once they have formed, either through continued planetesimal accretion, or through giant impacts. The migration of the core toward the inner cavity may actually place it in a region where planetesimal accretion is reduced due to increased velocity dispersion, and at present there have been no simulations of giant impact growth which include the gas disc. If a giant core can be formed, however, then it is likely that a gas giant planet will result. As it grows, a giant planet should leave the edge of the cavity and undergo type II migration (e.g. Lin \& Papaloizou 1993; Nelson et al. 2000), until it becomes trapped into the $4: 1$ resonance with the binary. This appears to be the most likely fate of a giant planet migrating in a circumbinary disc (Nelson 2003), although trapping is not necessarily permanent and simulations indicate a finite probablity of the planet being ejected from the system due to close encounters with the binary. The evolution of the planet during its growth from a core into a gas giant, however, has not been explored, and this will be the subject of a future paper.

Acknowledgements. The simulations performed in this paper were performed on the QMUL High Performance Computing facility purchased under the SRIF iniative.

\section{References}

Boss, A. P. 2006, ApJ, 641, 1148

Cresswell, P., \& Nelson, R. P. 2006, A\&A, 450, 833

Cresswell, P., Dirksen, G., Kley, W., \& Nelson, R. P. 2007, A\&A, 473, 329

Duquennoy, A., \& Mayor, M. 1991, A\&A, 248, 485

Dutrey, A., Guilloteau, S., \& Simon, M. 1994, A\&A, 286, 149

Eggenberger, A., Udry, S., \& Mayor, M. 2004, A\&A, 417, 353

Gladman, B. 1993, Icarus, 106, 247

Kley, W., Peitz, J., \& Bryden, G. 2004, A\&A, 414, 735

Lin, D. N. C., \& Papaloizou, J. C. B. 1993, Protostars and Planets III, 749

Marzari, F., \& Scholl, H. 2000, ApJ, 543, 328

Masset, F., \& Snellgrove, M. 2001, MNRAS, 320, L55

Masset, F. S., Morbidelli, A., Crida, A., \& Ferreira, J. 2006, ApJ, 642, 478

Moriwaki, K., \& Nakagawa, Y. 2004, ApJ, 609, 1065

Mugrauer, M., Neuhäuser, R., \& Mazeh, T. 2007, A\&A, 469, 755

Nelson, R. P. 2003, MNRAS, 345, 233

Nelson, R. P., Papaloizou, J. C. B., Masset, F., \& Kley, W. 2000, MNRAS, 318, 18

Papaloizou, J. C. B., \& Szuszkiewicz, E. 2005, MNRAS, 363, 153

Pierens, A., \& Nelson, R. P. 2007, A\&A, 472, 993

Press, W. H., Teukolsky, S. A., Vetterling, W. T., \& Flannery, B. P. 1992 (Cambridge: University Press), 2nd Ed.

Quintana, E. V., \& Lissauer, J. J. 2006, Icarus, 185, 1

Scholl, H., Marzari, F., \& Thébault, P. 2007, MNRAS, 380, 1119

Shakura, N. I., \& Sunyaev, R. A. 1973, A\&A, 24, 337

Sigurdsson, S., Richer, H. B., Hansen, B. M., Stairs, I. H., \& Thorsett, S. E. 2003 , Science, 301, 193

Stone, J. M., \& Norman, M. L. 1992, ApJS, 80, 753

Tanaka, H., \& Ward, W. R. 2004, ApJ, 602, 388

Thébault, P., Marzari, F., \& Scholl, H. 2006, Icarus, 183, 193

van Leer, B. 1977, J. Comput. Phys., 23, 276 